\title{
Aortic flow propagation velocity and neutrophil-to-lymphocyte ratio in coronary slow flow
}

\author{
Ozdemir $\mathrm{M}^{1}$, Asoglu $\mathrm{R}^{2}$, Aladag $\mathrm{N}^{3}$, Asoglu $\mathrm{E}^{4}$ \\ Adiyaman University Training and Research Hospital, Cardiology Department, Adiyaman, Turkey. \\ dr.asoglu@yahoo.com
}

\begin{abstract}
AIM: A useful tool for determining the presence of systemic inflammation is the neutrophil-to-lymphocyte ratio (NLR). Chronic inflammation causes more microvascular resistance, which is known to be involved with coronary slow flow (CSF). Aortic flow propagation velocity (APV) can be used to evaluate the aorta's rigidity. We hypothesized that NLR and APV might be related to CSF. Therefore, we aimed to evaluate the NLR and APV in CSF patients.

METHOD: Eighty-six CSF patients and 43 subjects with normal coronary flow were enrolled in this study. We utilized the Thrombolysis in Myocardial Infarction (TIMI) frame count (TFC) method for determining each enrolled patient's coronary flow rate. APV values were determined via color M-mode Doppler echocardiography. Differences in NLR and APV values were determined in CSF and control groups.

RESULTS: Patients with CSF had lower APV values and higher NLR values than the normal controls $(39.9 \pm 11.4$ vs $48.0 \pm 10.6, p<0.01 ; 3.3 \pm 2.8$ vs $2.3 \pm 1.2, p=0.03)$. TFC was significantly higher in CSF patients compared to controls $(29.8 \pm 5.0$ vs $12.9 \pm 2.6 ; p<0.01)$. TFC and APV were negatively correlated in CSF patients $(r=-0.338 p=0.001)$. APV was independently associated with CSF (OR: 1.164, Cl: 1.078-1.257, $p=0.001)$.

CONCLUSIONS: APV was significantly associated with TFC, and it is independently associated with CSF

(Tab. 4, Fig. 3, Ref. 30). Text in PDF www.elis.sk

KEY WORDS: coronary slow flow, neutrophil-to-lymphocyte ratio, aortic flow propagation velocity,

atherosclerosis, inflammation.
\end{abstract}

\section{Introduction}

Coronary slow flow (CSF) occurs as delayed coronary opacification in individuals that do not have obstructive CAD (coronary artery disease) (1). The TFC method is commonly utilized for the evaluation of CSF patients. TFC determines the amount of cine-angiographic frames it takes for the contrast to reach the distal arterial landmarks. The mechanism responsible for the CSF phenomenon has not been clearly elucidated; however, improperly functioning endothelial cells may be involved in its etiology (2). Moreover, it has been reported that there is a significant relationship between coronary flow rate and inflammatory markers (3).

Cardiovascular disease pathogenesis, including that of atherosclerosis, often involves inflammation (4). A clinical marker of inflammation is number of WBCs, while neutrophil-to-lymphocyte

${ }^{1}$ Kolan Hospital, Cardiology Department, Istanbul, Turkey, ${ }^{2}$ Adiyaman University Training and Research Hospital, Cardiology Department, Adiyaman, Turkey, ${ }^{3}$ Van Training and Research Hospital, Cardiology Department, Van, Turkey, and ${ }^{4}$ Mardin Community Hospital, Cardiology Department, Mardin, Turkey

Address for correspondence: Ramazan Asoglu, Dr, Adiyaman University Training and Research Hospital, Cardiology Department, Adiyaman, Turkey.

Phone: +90.5307763712 , Fax: +90.4162233842 ratio (NLR) is used for revealing cases with systemic inflammation (5). NLR can independently predict long-term death (6) in individuals who have undergone angioplasty. To calculate the NLR, divide neutrophils by lymphocytes. Interestingly, NLR is reported to be a predictor for the prognosis and severity of several cardiovascular diseases (7). In asymptomatic coronary artery disease (CAD) patients, there is an association between higher NLR with the disease's severity and complexity (8). As neutrophils indicate active inflammation, and because lymphocytes are protective and regulatory, the NLR indicates neutrophil/lymphocyte balance (9).

Atherosclerosis causes increased arterial resistance due to an increased thickness and stiffness in the arterial wall. A decreased speed of flow propagation in the central cavity of the artery may indicate a rise in the resistance of the aorta. A recently discovered echocardiographic parameter used to evaluate the stiffness of the descending thoracic aorta is colored M-mode-derived propagation rate (aorta flow propagation velocity-APV). It has been suggested that APV can indicate whether a patient has atherosclerotic heart disease. It has been reported that a good independent predictor of cardiac mortality and morbidity is aortic stiffness (10). A study by Gunes et al revealed a strong association between coronary atherosclerosis and APV (11). The results of that study are in accordance with others showing that APV is related to CAD (12). Since it has been published that increased NLR is associated with inflammation and atherosclerosis, we hypothesized that NLR and 

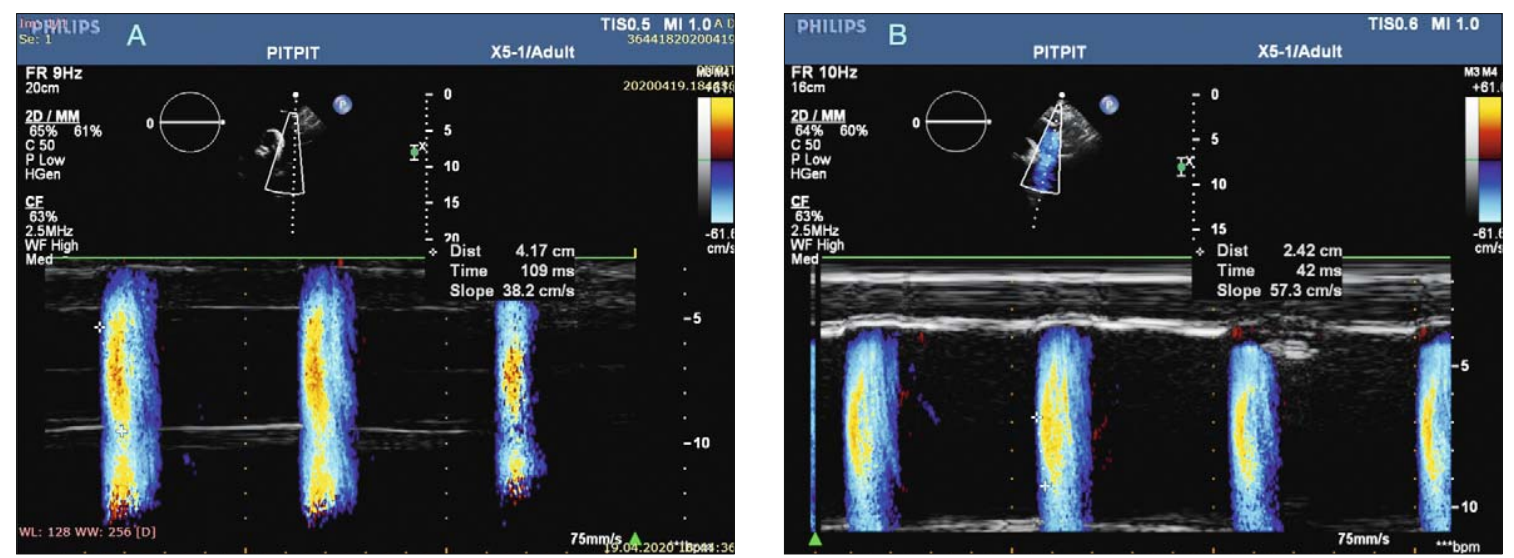

Fig. 1. Aortic velocity propagation $(\mathrm{AVP})$ in a CSF patient $(\mathrm{AVP}=38.2 \mathrm{~cm} / \mathrm{s})(\mathrm{Fig} .1 \mathrm{~A})$, and in a control subject $(\mathrm{AVP}=57.3 \mathrm{~cm} / \mathrm{s})$ (Fig. 1B). The AVP was calculated as explained in the main text.

APV might be related to CSF. Hence, the current study evaluated NLR and APV in individuals who had been diagnosed with CSF.

\section{Materials and methods}

From October 2014 to June 2015, 129 continuous individuals that had steady angina pectoris ( 86 with CSF, 43 with typical coronary flow) who underwent CAG in our institution were included in the current report. The individuals that were included in the current report were subjected to coronary angiography, which was completed via typical methods (Siemens Axiom Artis 2011, Siemens Healthcare, Erlangen, DE). All images were taken using normal views (i.e. left and right, caudal and cranial angulation). The angiogram for each patient was taken at a rate of 25 frames per second. All patients were injected with Iopromide for contrast (Ultravist-370, Bayer Schering Pharma, Germany). The patients enrolled in this study underwent coronary angiography because they were suspected of having CAD. A coronary artery was determined to be normal if it did not contain any lesions (obstructive or not). We determined coronary flow using TFC (13). Two cardiologists who had training in this area and did not know about any of the patient's clinical data evaluated the TFCs for every coronary vessel. The LCx and LAD arteriole TFCs were evaluated with angulation toward the foot in the right front stomach plane, and the right coronary arteries (RCA). TFC was determined with angulation toward the head in the left back stomach plane. For each study participant (CSF patient or normal control), the mean TFC was determined by determining the total sum of the TFCs of the $\mathrm{LCx}, \mathrm{LAD}$, and RCA and dividing this number by 3 . If a patient's vessel had a TFC value more than 2 SD from what is considered normal, the patient was determined to have CSF (13).

Each of the included patients in this study (CSF patients and controls) underwent a rigorous physical examination, blood analysis, and echocardiography to determine if they had a structural heart disease. Exclusion criteria were anemia, uncontrolled hypertension or diabetes mellitus, dysfunction in the left ventricle (LVEF $<50 \%$ ) or dysplasia, modest or serious stiffening of the valve or vomiting, irregular heartbeat, aortic aneurysms, ACS, known malignancy, problems with the thyroid, hepatic or kidney dysfunction, systemic or local infection, inflammatory diseases, and if the patient was taking a medication that may interfere with the NLR measurement.

A specialist who was unaware of the patient's clinical data performed all of the echocardiography done in this study. Echocardiography was completed while the individual was in the left lateral dorsal stance at rest with a Vivid S6 (GE, Horton, Norway) coupled to a 3.0 MHz transducer. The parasternal extended Mmode view mode was used to determine LVESD and LVEDD. In addition, we measured the girth of the IVS and the PW, left atrium diameter, and ejection fraction. Doppler echocardiography was used to determine mitral A, mitral E, DT and IVRT. With the subject on his/her back, all color M-mode Doppler recordings were taken from the suprasternal window. Importantly, the indicator was in a position that was side by side with the direction of chief flow in the descendant aorta. We had a Nyquist limit that was between thirty and fifty centimeters per second, and then we went to M-mode that had a recording interval of two hundred millimeters per second. In cases where the frame slope was not easily defined, we used baseline shifting to alter the aliasing velocity. Next, we calculated APV as follows: the space in the middle of the points (at start and finish of the slope) was divided by the period in the middle of these places. Hence, APV reveals the flow rate downward of the artery. Each APV value presented herein represents the average value of three or more recordings (Fig. 1). The APV intra- and inter-observer variability was excellent (correlation coefficients of 0.92 and 0.90 , respectively).

Following an overnight fast of at least 8 hours, atraumatic puncture was used to draw blood from each patient's antecubital vein into tubes containing seven and two-tenths milligrams of tripotassium EDTA. The blood from each patient was analyzed immediately via an automatic blood counter. An LH 780 machine (Beckman Coulter Inc, Miami, FL) was used to determine each blood sample's hematological parameters (e.g., numbers of neutrophils, lymphocytes, and WBCs). Neutrophils were divided by 
Tab. 1. The demographic and clinical data of the study population.

\begin{tabular}{lccc}
\hline & $\begin{array}{c}\text { CSF } \\
(\mathrm{n}=86)\end{array}$ & $\begin{array}{c}\text { Control } \\
(\mathrm{n}=43)\end{array}$ & $\mathrm{p}$ \\
\hline Age (years) & $49.4 \pm 10.1$ & $46.0 \pm 8.7$ & 0.16 \\
Male \%(n) & $66(38)$ & $33(19)$ & 0.95 \\
Hypertension \%(n) & $68(28)$ & $31(13)$ & 0.78 \\
Diabetes mellitus \%(n) & $53(15)$ & $46(13)$ & 0.09 \\
Smoking \%(n) & $56(40)$ & $43(31)$ & $\mathbf{0 . 0 0 6}$ \\
Systolic BP (mm Hg) & $122.9 \pm 14.2$ & $121.4 \pm 14.5$ & 0.56 \\
Diastolic BP (mm Hg) & $76.8 \pm 8.2$ & $72.0 \pm 9.1$ & 0.06 \\
Ejection fraction $(\%)$ & $56.4 \pm 3.8$ & $56.3 \pm 3.8$ & 0.82 \\
Heart rate (bpm) & $76.8 \pm 9.4$ & $76.1 \pm 10.2$ & 0.70 \\
White blood cell count $\left(103 / \mathrm{mm}^{3}\right)$ & $7.9 \pm 1.6$ & $7.2 \pm 2.0$ & $\mathbf{0 . 0 4}$ \\
Neutrophil $\left(103 / \mathrm{mm}^{3}\right)$ & $6.2 \pm 0.9$ & $5.9 \pm 0.8$ & 0.06 \\
Lymphocyte $\left(103 / \mathrm{mm}^{3}\right)$ & $2.4 \pm 0.8$ & $2.8 \pm 0.7$ & $\mathbf{0 . 0 1}$ \\
NLR n & $3.3 \pm 2.8$ & $2.3 \pm 1.2$ & $\mathbf{0 . 0 3}$ \\
Descending aorta diameter $(\mathrm{cm})$ & $2.9 \pm 0.2$ & $2.7 \pm 0.2$ & 0.10 \\
Left atrial diameter $(\mathrm{cm})$ & $3.6 \pm 0.3$ & $3.5 \pm 0.4$ & 0.13 \\
IVS (cm) & $1.06 \pm 0.14$ & $1.03 \pm 0.11$ & 0.20 \\
PWD (cm) & $1.00 \pm 0.11$ & $0.96 \pm 0.10$ & 0.11 \\
LVEDD (cm) & $4.7 \pm 0.3$ & $4.7 \pm 0.4$ & 0.88 \\
LVESD (cm) & $3.3 \pm 0.3$ & $3.2 \pm 0.3$ & 0.64 \\
Mitral E (m/sec) & $0.6 \pm 0.1$ & $0.6 \pm 0.1$ & 0.09 \\
Mitral A (m/sec) & $0.5 \pm 0.1$ & $0.4 \pm 0.1$ & 0.06 \\
DT (msec) & $190.8 \pm 23.0$ & $186.0 \pm 19.1$ & 0.06 \\
IVRT (msec) & $84.9 \pm 5.6$ & $81.6 \pm 5.2$ & 0.08 \\
APV & $39.9 \pm 11.4$ & $48.0 \pm 10.6$ & $<\mathbf{0 . 0 1}$ \\
\hline CSF - Cory & & & \\
& & &
\end{tabular}

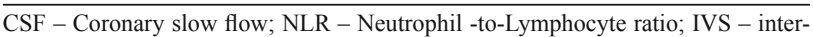
ventricular septum; PWD - posterior wall diameter; LVEDD - left ventricular enddiastolic diameter; LVESD - left ventricular end-systolic diameter; Mitral E - early diastolic mitral flow velocity; Mitral A - late diastolic mitral flow velocity; DT deceleration time; IVRT - isovolumic relaxation time; APV - Aortic propagation velocity. $p$ value was calculated by the student's t test or Chi-square test. Values are mean \pm SD or median [25th, 75th] and number (percentage). $\mathrm{p}<0.05$ was considered statistically significant

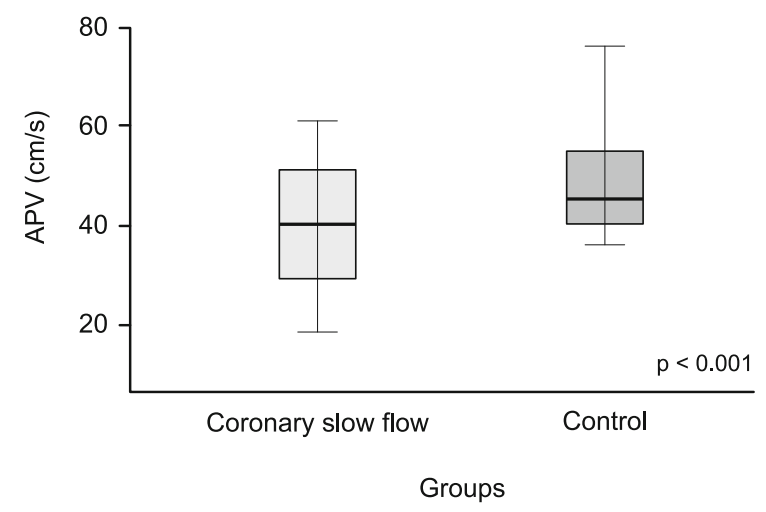

Fig. 2. Aortic flow propagation velocity (APV) values between groups.

lymphocytes to obtain NLR. Each patent's demographic data, history of cardiovascular disease, and any CSF risk factors were recorded from his/her medical records. During the coronary angiography procedure, we also recorded blood pressure (systolic and diastolic) and heart rate. Patients were considered to have diabetes if their fasting blood sugar was $\geq 126 \mathrm{mg} / \mathrm{dL}$ or if they were currently using a prescription to treat diabetes. Those who had systolic $\mathrm{BP} \geq 140 \mathrm{~mm} \mathrm{Hg}$, diastolic $\mathrm{BP} \geq 90 \mathrm{~mm} \mathrm{Hg}$, or were presently using a prescription to treat elevated $\mathrm{BP}$ were considered
Tab. 2. Angiographic data of the study population.

\begin{tabular}{lccl}
\hline & CSF $(\mathrm{n}=86)$ & Control $(\mathrm{n}=43)$ & $\mathrm{p}$ \\
\hline LAD-TFC & $43.7 \pm 7.4$ & $15.8 \pm 3.4$ & $<0.01$ \\
CX-TFC & $24.7 \pm 7.6$ & $12.3 \pm 3.0$ & $<0.01$ \\
RCA-TFC & $21.1 \pm 4.6$ & $10.7 \pm 2.7$ & $<0.01$ \\
Mean TFC & $29.8 \pm 5.0$ & $12.9 \pm 2.6$ & $<0.01$ \\
Total & $89.5 \pm 15.0$ & $38.9 \pm 8.0$ & $<0.01$ \\
LAD \% (n) & $95(82)$ & & \\
CX \% (n) & $16(14)$ & & \\
RCA \% (n) & $22(19)$ & & \\
One vessel \% (n) & $72(62)$ & & \\
Two vessels \% (n) & $22(19)$ & & \\
Three vessels \% (n) & $6(5)$ & & \\
\hline
\end{tabular}

CSF - Coronary slow flow; TFC - TIMI frame count; LAD - Left anterior descending Artery; CX -Circumflex artery; RCA - Right coronary artery. p value was calculated by the student's $t$ test. $p<0.05$ was considered statistically significant

to have hypertension. Patients were considered to be smokers if they actively smoked or had smoked in the past ( $>10$ pack-years). Each included individual had 12-lead electrocardiography (ECG) performed. This study was okayed by Van Training and Research Hospital's ethics committee, and every participant included provided consent prior to participating.

\section{Analyzing the data}

All the data collected in this study was analyzed with SPSS software (v. 20.0 for Windows, SPSS Inc, Chicago, IL). To determine whether continuous variables had a normal distribution, we utilized the Kolmogorov-Smirnov test. Throughout this paper, all variables that were normally distributed are given as averages \pm standard deviations (SD), while any categorical variables are given as percentages. If parameters were normally distributed, a Student's unpaired t-test determined any differences. A chi-square test or Fisher's exact test compared occurrences of nominal variables. Correlation analyses were performed via Pearson tests. Any independent predictors of CSF were determined via the multivariate logistic regression model. We considered $p<0.05$ as significant.

\section{Results}

Table 1 reveals all study participants' clinical and demographic data. Sex and age were not very different among the groups, nor were there any differences in diabetes mellitus, HR, hypertension, and BP (systolic and diastolic). The CSF group had significantly more smokers than the normal control group. Regarding hematologic parameters, neutrophil count was similar among the CSF and normal control group, while WBC count and NLR were much greater in the CSF versus normal patients, and lymphocyte count was much lower. In echocardiographic analyses, ejection fraction, mitral E velocity, mitral A velocity, DT, IVRT, IVS diameter, PW diameter, LVEDD, and LVESD did not show any differences between the CSF versus normal patients. On the contrary, CSF patients had significantly lower APV values (Fig. 2). In the angiographic analyses, the CSF patients had significantly higher TFC (Tab. 2). Table 3 presents the correlation analyses between the Mean-TFC and age, WBC, neutrophil, lymphocyte, NLR, 
Tab. 3. Correlation analyzes between the Mean-TFC and age, white blood cell count, neutrophil, lymphocyte, neutrophil-to-lymphocyte ratio and aortic propagation velocity.

\begin{tabular}{lcc}
\hline & $\mathrm{r}$ & $\mathrm{p}$ \\
\hline Age & 0.170 & 0.054 \\
White blood cell count & 0.101 & 0.257 \\
Neutrophil & 0.104 & 0.242 \\
Lymphocyte & -0.179 & $\mathbf{0 , 0 4 3}$ \\
Neutrophil-to-lymphocyte ratio & 0.147 & 0.096 \\
Aortic propagation velocity & -0.338 & $\mathbf{0 . 0 0 1}$ \\
\hline
\end{tabular}

Mean-TFC - Mean TIMI frame counts. Pearson test was used to analyze the relationship between Mean-TFC and study variables where appropriate. correlation coefficient (r). $\mathrm{p}<0.05$ was considered statistically significant

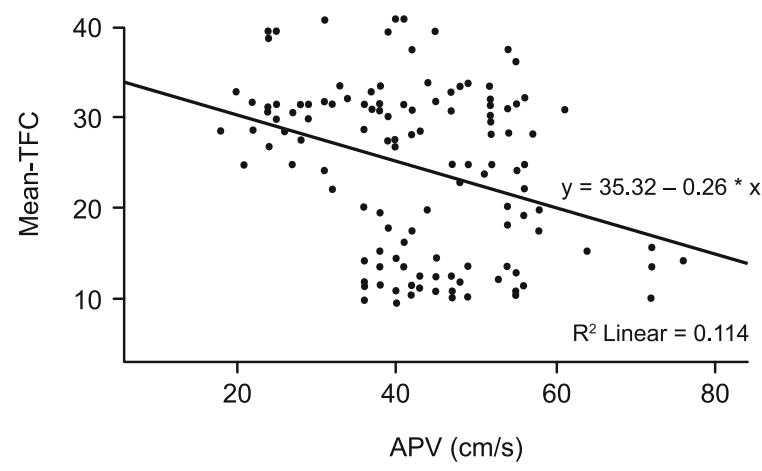

Fig. 3. The correlation between Mean-TFC (TIMI-frame counts) and Aortic flow propagation velocity $(\mathrm{APV})$ values $(\mathrm{r}=-0.338 \mathrm{p}=0.001)$.

and APV. Our analyses indicate a significant negative correlation among Mean-TFC and lymphocyte and APV $(\mathrm{r}=-0.179, \mathrm{p}=$ 0,$043 ; \mathrm{r}=-0.338, \mathrm{p}=0.001$; respectively). Figure 3 shows a correlation among the Mean-TFC and APV $(r=-0.338, \mathrm{p}=0.001)$. Table 4 reveals associations among CSF and several parameters. These analyses revealed that APV is a lone prognosticator of CSF (OR: 1.164, CI: $1.078-1.257, \mathrm{p}=0.001$ ).

\section{Discussion}

Our current study revealed three important results for CSF patients. First, CSF patients had significantly higher NLR and significantly lower APV than the normal controls. Second, we found a significant negative correlation between TFC and APV values. Third, we discovered that APV is an independent predictor for CSF. Taken together, these results submit that increased serum NLR and lower APV values may play a role in the dysregulation of endothelial cells in CSF, inflammation, and atherosclerotic process.

NLR is a marker of inflammation that is not difficult to calculate, and is easy to obtain (14). The relationship between atherosclerosis and NLR has not been clearly elucidated. NLR has been used to show the inflammatory pathogenesis and severity of CAD in various studies (15). One study reported that elevated NLR could be used to predict atherosclerotic progression (16), and it was presented as a better indicator of adverse heart problems than the gap in leukocytes (6). Recently, it has been shown that NLR can be
Tab. 4. Multivariate logistic regression analysis to determine the independent predictors of Coronary slow flow.

\begin{tabular}{lcc}
\hline & \multicolumn{2}{c}{ Multivariate } \\
\cline { 2 - 3 } & OR $(95 \% \mathrm{CI})$ & $\mathrm{p}$ \\
\hline NLR & $1.025(0.603-1.742)$ & 0.929 \\
APV & $1.164(1.078-1.257)$ & $\mathbf{0 . 0 0 1}$ \\
Neutrophil & $0.492(0.175-1.377)$ & 0.177 \\
Lymphocyte & $3,313(0.784-14.001$ & 0.103 \\
Age & $0.964(0.920-1.010)$ & 0.125 \\
Diabetes Mellitus & $0.404(0.127-1.281)$ & 0.124 \\
Smoking & $2.381(0.914-6.204)$ & 0.076 \\
\hline NLR - Neutrophil-to-lymphocyte ratio; APV - Aortic propagation velocity. OR -
\end{tabular}
odds ratio; $\mathrm{CI}$ - confidence interval; Statistical significance $(\mathrm{p}<0.05)$

important for the prognosis of many cardiac conditions. Preprocedural NLR has been reported to be a good independent predictor of the no-flow phenomenon in people known to have STEMI (STelevation myocardial infarction) (17). Akyel et al's study revealed that people known to have hemodynamically significant coronary artery stenosis had significantly higher NLR. Moreover, the authors revealed that NLR was an independent predictor of this condition as determined by fractional flow reserve (18). Another related study determined the CAD's complexity with a SYNTAX score, and results of that study revealed NLR was a lone prognosticator of CAD's complexity as well as presence (19).

The pathophysiology of CSF has not been fully elucidated, but one possibility includes endothelial dysfunction and relationship to atherosclerosis. It has been shown that there is a close relationship between endothelial dysfunction and inflammatory parameters (20). We know that increased inflammatory parameters harm the endothelium and can lead to endothelial dysfunction (21). A previous study indicated that inflammatory parameters are increased in patients with CSF (22). Dogan et al evaluated the association among NLR and slow coronary flow CSF. Data from that study revealed that NLR is associated with the occurrence of CSF, but not its extent. The study by Dogan et al also revealed that NLR is a good independent predictor of CSF (23). In our current study, we hypothesized that those individuals with CSF would have inflammation associated with atherosclerosis and endothelial dysfunction. One study investigated endothelial function in CSF patients, and found that CSF patients have impaired endothelial function, and an important association among endothelial dysfunction and TFC (2). Çetin et al investigated the ability of NLR and plateletto-lymphocyte ratio to predict disease presence and severity in CSF patients. In their study, $78 \mathrm{CSF}$ and 50 normal coronary flow controls were enrolled, and it was determined that the individuals with CSF had much greater NLR. The same study reported an important positive correlation among NLR and TFC (24). In parallel to that published study, our current study also determined that NLR had a significant positive correlation with mean-TFC. Moreover, we presented that NLR was higher in CSF patients. Similarly to what is published in the literature, data from our current study also revealed that CSF is related to inflammation.

The first stage of atherosclerosis is endothelial dysfunction. As atherosclerosis progresses, there are increases in the thickness of 
the arterial wall and in the aorta's stiffness, while there are decreases in aortic strain (AS) and aortic distensibility (AD) (25). Arterial stiffness significantly increases the risk for developing atherosclerosis, and it is also connected to heart disease risk factors, such as CAD (26). APV is a straightforward echocardiography parameter that can be used during routine physical exams. APV can also be used to determine those that are at high-risk for CAD. APV flow can be decreased by increased arterial resistance. A report by Gunes et al revealed that APV is CAD's most important predictor (11). Sen et al evaluated APV in CAD patients. That study included 51 CAD patients and 42 healthy controls (normal coronary arteries). Results of that study indicate that the CAD patients had significantly lower APV, AD, and AS than the healthy controls. There was a statistically significant relation between $\mathrm{APV}, \mathrm{AS}$, and $\mathrm{AD}$ in that study (27). Additionally, Chetty et al determined the association among APV and atherosclerotic CAD burden (as determined via SYNTAX score). They demonstrated an inverse correlation between APV and CAD severity. Moreover, they showed that APV had an inverse and robust correlation with the SYNTAX score (28).

Simsek et al investigated the role of APV as a way to determine endothelial dysfunction in CSF patients. They determined APV in those with CSF $(n=90)$ as well as in those with normal coronary arteries (NCA, $n=39$ ), and found that those with CSF had significantly lower APF than NCA participants. In addition, they demonstrated that APV is an easy, sensible method that is highly associated with CSF (29). Similarly, we found that APV was decreased a lot in individuals with CSF. Also, we presented an important negative association among Mean-TFC and APV $(\mathrm{r}=-0.338, \mathrm{p}=0.001)$. Yaman et al assessed the early and late APV values in STEMI patients and evaluated their relationship with NLR. They reported that there were negative and significant correlations between APV and NLR at admission $(\mathrm{r}=-0.61, \mathrm{p}<$ $0.01)$ and at a 3-month follow-up $(\mathrm{r}=-0.59, \mathrm{p}<0.01)$ in patients diagnosed with STEMI. Increments in APV values were correlated with decrements in NLR. APV might be a novel biomarker as a reactant in individuals diagnosed with AMI. Hence, the authors of that report considered that APV was an important echocardiographic marker for the presence of inflammation (30). Currently, we revealed that APV was very much decreased in CSF patients versus normal healthy controls. Moreover, APV had a significant negative correlation with TFC, and it was independently associated with CSF. Similarly to the literature, we propose that APV might be an echocardiographic parameter that can be used as an indicator for the presence of inflammation in patients with CSF.

The present study provides invaluable results regarding CSF patients, but is not without limitations. First, this study included a low number of participants, and was performed at a single hospital. In addition, we did not measure any other known inflammatory indicators (e.g., IL-6, TNF- $\alpha$, or CRP). Further, the APV and NLR were only measured once for each patient (at the time of admission), so we could not determine whether medical treatment had any effect on these values. As intravascular ultrasound is not routinely performed in our hospital, we did not perform it in CSF patients in this study. Therefore, we suggest that further studies including a much larger patient population from different centers are required in order to more accurately define the relationship between NLR and APV and CSF.

\section{Learning points}

- Aorta flow propagation velocity (APV) is used to evaluate the stiffness of the descending thoracic aorta via M-mode echocardiography.

- increased neutrophil-to-lymphocyte ratio (NLR) is associated with inflammation and atherosclerosis.

- Patients with coronary slow flow (CSF) have significantly higher NLR and lower APV values than subjects who have normal coronary arteries.

- The relation between CSF and higher NLR and lower APV implies that endothelial dysfunction might be purposeful in CSF's etiopathogenesis.

\section{Conclusions}

In conclusion, results of the current study revealed that CSF patients have significantly higher NLR and significantly lower APV than patients who have normal coronary arteries. CSF individuals had an APV that was greatly associated with TFC. The relation between CSF and higher NLR and lower APV implies that endothelial dysfunction and having atherosclerosis may be purposeful in CSF's etiopathogenesis. Others must perform studies so that we can more clearly define the pathophysiologic role of NLR and APV in CSF.

\section{References}

1. Tambe AA, Demany MA, Zimmerman HA, Mascarenhas E. Angina pectoris and slow flow velocity of dye in coronary arteries--a new angiographic finding. Am Heart J 1972; 84 (1): 66-71.

2. Sezgin AT, Sgrc A, Barutcu I et al. Vascular endothelial function in patients with slow coronary flow. Coronary Artery Dis 2003; 14 (2): 155-161.

3. Kalay N, Aytekin M, Kaya MG et al. The relationship between inflammation and slow coronary flow: increased red cell distribution width and serum uric acid levels. Arch Turk Soc Cardiol 2011; 39 (6): 463-468.

4. Libby P, Ridker PM, Maseri A. Inflammation and atherosclerosis. Circulation 2002; 105 (9): 1135-1143.

5. Zahorec R. Ratio of neutrophil to lymphocyte counts-rapid and simple parameter of systemic inflammation and stress in critically ill. Bratisl Med J 2001; 102 (1): 5-14.

6. Duffy BK, Gurm HS, Rajagopal V, Gupta R, Ellis SG, Bhatt DL. Usefulness of an elevated neutrophil to lymphocyte ratio in predicting longterm mortality after percutaneous coronary intervention. Amer J Cardiol 2006; 97 (7): 993-996.

7. Bhat T, Teli S, Rijal J et al. Neutrophil to lymphocyte ratio and cardiovascular diseases: a review. Expert Rev Cardiovasc Ther 2013; 11 (1): 55-59.

8. Açar G, Fidan S, Uslu ZA et al. Relationship of neutrophil-lymphocyte ratio with the presence, severity, and extent of coronary atherosclerosis detected by coronary computed tomography angiography. Angiology 2015; 66 (2): 174-179. 
513-518

9. Bhutta H, Agha R, Wong J, Tang TY, Wilson YG, Walsh SR. Neutrophil-lymphocyte ratio predicts medium-term survival following elective major vascular surgery: a cross-sectional study. Vasc Endovasc Surg 2011; 45 (3): 227-231.

10. Arnett DK, Evans GW, Riley WA. Arterial stiffness: a new cardiovascular risk factor? Amer J Epidemiol 1994; 140 (8): 669-682.

11. Güneş Y, Tuncer M, Yildirim M, Güntekin U, Gümrükçüoğlu HA, Sahin M. A novel echocardiographic method for the prediction of coronary artery disease. Med Sci Monit 2008; 14 (9): MT42-46.

12. Cohn JN, Finkelstein S, McVeigh G et al. Noninvasive pulse wave analysis for the early detection of vascular disease. Hypertension 1995; 26 (3): 503-508.

13. Gibson CM, Cannon CP, Daley WL et al. TIMI frame count: a quantitative method of assessing coronary artery flow. Circulation 1996 Mar 1; 93 (5): 879-888.

14. Karaman M, Balta S, AY SA et al. The comparative effects of valsartan and amlodipine on vWf levels and N/L ratio in patients with newly diagnosed hypertension. Clin Exp Hypertension 2013; 35 (7): $516-522$

15. Arbel Y, Finkelstein A, Halkin A et al. Neutrophil/lymphocyte ratio is related to the severity of coronary artery disease and clinical outcome in patients undergoing angiography. Atherosclerosis 2012; 225 (2): 456-460.

16. Kalay N, Dogdu O, Koc F et al. Hematologic parameters and angiographic progression of coronary atherosclerosis. Angiology 2012; 63 (3): 213-217.

17. Yildiz A, Yuksel M, Oylumlu M et al. The utility of the plateletlymphocyte ratio for predicting no reflow in patients with st-segment elevation myocardial infarction. Clin Appl Thromb Hemostasis 2015; 21 (3): 223-228.

18. Akyel A, Yayla Ç, Erat M et al. Neutrophil-to-lymphocyte ratio predicts hemodynamic significance of coronary artery stenosis. Anatol J Cardiol 2016; 15 (12): 1002 .

19. Sönmez O, Ertaş G, Bacaksiz A et al. Relation of neutrophil-tolymphocyte ratio with the presence and complexity of coronary artery disease: an observational study. Anadolu Kardiyol Derg 2013; 13 (7): $662-667$.
20. Antoniades C, Tousoulis D, Vasiliadou C et al. Combined effects of smoking and hypercholesterolemia on inflammatory process, thrombosis/ fibrinolysis system, and forearm hyperemic response. Amer J Cardiol 2004 Nov 1; 94 (9): 1181-1184.

21. Devaraj S, Yun J-M, Adamson G, Galvez J, Jialal I. C-reactive protein impairs the endothelial glycocalyx resulting in endothelial dysfunction. Cardiovascular research 2009; 84 (3): 479-84.

22. Li J-J, Qin X-W, Li Z-C et al. Increased plasma C-reactive protein and interleukin- 6 concentrations in patients with slow coronary flow. Clin Chim Acta 2007; 385 (1): 43-47.

23. Doğan M, Akyel A, Çimen T et al. Relationship between neutrophil to lymphocyte ratio and slow coronary flow. Clin Appl Thromb Hemostasis $2015 ; 21$ (3): $251-425$.

24. Çetin M, Kiziltunc E, Elalmiş ÖU et al. Predictive value of neutrophil lymphocyte ratio and platelet lymphocyte ratio in patients with coronary slow flow. Acta Cardiol Sin 2016; 32 (3): 307.

25. Park SM, Seo HS, Lim HE et al. Assessment of arterial stiffness index as a clinical parameter for atherosclerotic coronary artery disease. Circulat J 2005; 69 (10): 1218-1222.

26. Franklin SS, Larson MG, Khan SA et al. Does the relation of blood pressure to coronary heart disease risk change with aging? The Framingham Heart Study. Circulation 2001; 103 (9): 1245-1249.

27. Sen T, Tufekcioglu O, Ozdemir M et al. A new echocardiographic parameter of aortic stiffness and atherosclerosis in patients with coronary artery disease: aortic propagation velocity. J Cardiol 2013; 62 (4): 236-240.

28. Chetty PV, Rajasekhar D, Vanajakshamma V, Ranganayakulu KP, Chaithanya DK. Aortic velocity propagation: a novel echocardiographic method in predicting atherosclerotic coronary artery disease burden. J Saudi Heart Assoc 2017; 29 (3): 1761-84.

29. Simsek H, Sahin M, Gunes Y et al. A novel echocardiographic method as an indicator of endothelial dysfunction in patients with coronary slow flow. Eur Rev Med Pharmacol Sci 2013; 17 (5): 689-693.

30. Yaman M, Arslan U, Beton O, Pamukcu H, Dogdu O. Early and late aortic propagation velocity values in STEMI patients after successful primary PCI and their relationship with neutrophil to lymphocyte ratio. Eur Rev Med Pharmacol Sci 2016; 20: 912-918.

Received May 28, 2020. Accepted January 27, 2021. 